\title{
NOUVELLe
}

\section{Activation de la voie Notch par OTT-MAL dans les leucémies aiguës mégacaryoblastiques}

Olivier A. Bernard, D. Gary Gilliland, Thomas Mercher
Inserm EMI0210, Université Paris Descartes,

Hôpital Necker, Tour Pasteur,

149 rue de Sèvres, 75015 Paris, France.

thomas.mercher@inserm.fr
> Les leucémies aiguës résultent de l'accumulation de mutations dans des cellules souches ou des progéniteurs hématopoïétiques conduisant à une augmentation de leur survie, à une prolifération et à un blocage de différenciation. Elles peuvent être classées en fonction de la lignée hématopoïétique préférentiellement atteinte. Il est généralement admis que la transformation nécessite la coopération d'au moins deux évènements oncogéniques. Dans les leucémies aiguës, les mutations touchant les facteurs de transcription altèrent généralement la différenciation des progéniteurs hématopoïétiques et coopèrent fréquemment avec des mutations touchant des intermédiaires de voies de signalisation intracellulaires qui stimulent la survie et la prolifération cellulaires.

\section{La diversité des leucémies aiguës} myéloïdes à mégacaryoblastes Les bases moléculaires des leucémies aiguës myéloïdes (LAM) à mégacaryoblastes (LAM7 dans la classification $F A B^{1}$ ) sont mal connues. Elles se présentent selon une distribution bimodale selon l'âge [1]. Les anomalies chromosomiques retrouvées chez l'adulte sont, en règle générale, diverses et non spécifiques $[1,2]$. Chez l'enfant, deux sous-groupes sont identifiés: les LAM7 associées à la translocation $t(1 ; 22)(p 13 ; q 13)$ chez le nourrisson et le jeune enfant et celles qui se développent

${ }^{1}$ Proposée en 1982 par le groupe FAB (French American British), cette classification se fonde sur le nombre de blastes et la morphologie des cellules observées sur le myélogramme. Elle distingue neuf classes de LAM : LAMO à LAM7. chez les patients porteurs de trisomie 21 constitutionnelle (Down syndrome). Ce dernier groupe se caractérise par des mutations du gène codant pour le facteur de transcription GATA-1, qui est normalement impliqué dans le contrôle de la différenciation érythro-mégacaryocytaire [3]. Des analyses chez I'homme et chez la souris indiquent que, bien que des anomalies de la mégacaryopoïèse soient observées au cours du développement chez les patients trisomiques, les mutations GATA-l ne sont pas suffisantes pour induire une transformation leucémique, soulignant la nécessité de mutations additionnelles pour le développement de ce type de LAM7.

\section{Coopération entre 0TT-MAL} et l'activation de la signalisation du récepteur de la thrombopoiétine La translocation $t(1 ; 22)$ conduit à la fusion (OTT-MAL, one twenty-twomegakaryocytic acute leukemia) de deux gènes OTT/RBMI5 et MAL/MKLI codant pour des facteurs de transcription [4]. OTT est un facteur pléiotrope de l'hématopoïèse [5] et a été impliqué dans le contrôle de l'activité de RBPJ (recombination signal binding protein for immunoglobulin $\kappa$ J region) [6], un acteur majeur de la réponse transcriptionnelle à la voie de signalisation Notch. MAL est un cofacteur du SRF (serum response factor) qui est impliqué dans la réponse à la voie de signalisation Rho GTPase/G-actine [7]. Nous avons modélisé l'expression de cet oncogène chez la souris en réalisant un knock-in de I'ADN complémentaire de
MAL au locus Ott murin [8]. L'analyse de ce modèle montre que l'expression d'OTT-MAL induit des anomalies de différenciation des cellules souches hématopoïétiques qui s'engagent vers la lignée mégacaryocytaire. De plus, ces animaux développent des proliférations hématopoiétiques et des leucémies aiguës, présentant les caractéristiques des LAM7 humaines, mais avec une faible pénétrance (1/10 animaux) et une latence longue (> 18 mois). À partir d'un animal leucémique, une lignée cellulaire dépendante pour sa prolifération de la cytokine SCF (stem cell factor) et exprimant fortement OTT-MAL a été obtenue. Nous avons utilisé cette lignée pour tester l'hypothèse de la nécessaire coopération oncogénique dans l'émergence d'une leucémie: nous avons exprimé différentes protéines de signalisation mutées, choisies parmi celles qui sont naturellement observées dans des hémopathies mégacaryoblastiques humaines. Nous avons montré que l'expression d'une mutation du récepteur MPL (le récepteur de la thrombopoïétine, cytokine clé de la différenciation mégacaryocytaire), MPL ${ }^{W 515 L}$ - mais pas celle d'autres mutants dont FLT3 ${ }^{\text {ITDN51, }}$ JAK2 ${ }^{\text {V617F }}$, JAK2 $2^{\text {T875N }}$ - entraîne une levée de la dépendance au SCF de la lignée, associée à une activation spécifique de la phosphorylation des kinases $\varepsilon R K 1 / 2$, des intermédiaires de la voie MAPK impliquée dans le développement mégacaryocytaire. De plus, alors que l'expression de MPL ${ }^{W 515 L}$ dans des cellules sauvages greffées à des receveurs primaires normaux induit des syndromes myéloprolifératifs non transplantables à 
des animaux receveurs secondaires, la transduction de cet ADNc muté MPL ${ }^{W 515 L}$ dans un contexte OTT-MAL induit des leucémies mégacaryoblastiques aiguës transplantables, avec une pénétrance complète. Les souris exprimant OTT-MAL et MPL ${ }^{W 515 L}$ montrent une amplification des progéniteurs mégacaryocytaires, un blocage de la différenciation, ce qu'indique une réduction importante de la ploïdie, une thrombocytopénie et une fibrose médullaire et splénique, des caractéristiques retrouvées chez les patients atteints de LAM7.

\section{OTT-MAL interfère}

\section{avec la voie de signalisation Notch}

Sur un plan mécanistique, nous avons montré que la protéine OTT-MAL interagit directement avec RBPJ et active de façon anormale la transcription des gènes cibles de RBPJ. L'expression d'un mutant dominant négatif de RBPJ, ne pouvant plus lier I'ADN, inhibe la prolifération des cellules de la lignée cellulaire exprimant OTTMAL. Enfin, l'analyse du profil transcriptionnel d'échantillons de patients ayant une LAM7 et porteurs d'une $t(1 ; 22)(p 13 ; q 13)$ montre une activation de la voie Notch qui n'est pas retrouvée dans les échantillons de cellules issus d'individus porteurs d'une LAM7 dans un contexte de trisomie 21. Sur la base de ces observations et des résultats récents indiquant le rôle positif de la voie Notch dans la spécification du lignage mégacaryocytaire [9], il est donc vraisemblable que la dérégulation par OTT-MAL de la transcription des gènes cibles de la voie Notch soit responsable de l'association spécifique des $t(1 ; 22)(p 13 ; q 13)$ avec les leucémies à mégacaryoblastes.

La voie Notch joue également un rôle positif important dans l'engagement des cellules souches hématopoïétiques vers la lignée lymphocytaire T [10]. De plus, des mutations activatrices du récepteur Notchl sont retrouvées dans plus de $50 \%$ des leucémies aiguës lymphoblastiques T chez l'homme [11]. Nos observations montrent de façon inattendue l'implication d'une voie de signalisation commune entre les deux lignages, mégacaryocytaire et lymphoïde T. Nous

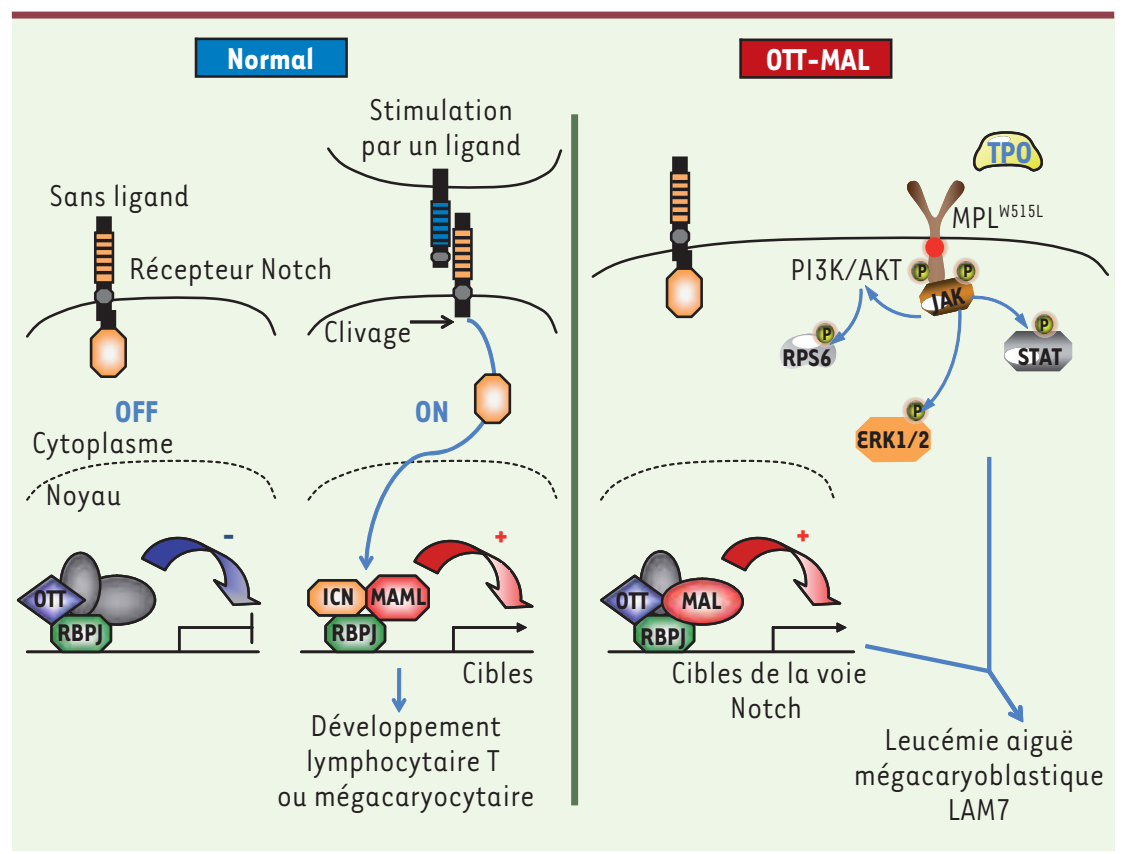

cherchons maintenant à comprendre les spécificités de la voie Notch lorsqu'elle intervient dans la différenciation normale des cellules souches hématopoïétiques vers la lignée mégacaryocytaire [12] ou lymphocytaire $T$ ainsi que dans la transformation tumorale mégacaryoblastique.

Dans l'ensemble, l'étude fonctionnelle de la translocation chromosomique $t(1 ; 22)(p 13 ; q 13)$ à l'aide d'un modèle reproduisant l'anomalie observée chez I'homme a permis de révéler un mécanisme de transformation nouveau dans les leucémies aiguës à mégacaryoblastes. En effet, ces résultats indiquent une coopération oncogénique entre une activation anormale des gènes cibles de la voie Notch par OTT-MAL et une activation constitutive du récepteur MPL dans la transformation mégacaryocytaire (Figure 1). II est maintenant important de rechercher une altération de la régulation des gènes cibles de la voie Notch dans les autres échantillons de LAM7. $\diamond$

The 0TT-MAL fusion oncogene: another Notch in megakaryoblastic leukemia

Figure 1. Modèle de transformation mégacaryoblastique par l'oncogène de fusion OTT-MAL. Dans un contexte normal, la liaison du récepteur Notch par un de ses ligands entraîne un clivage du récepteur conduisant à la migration de la partie intra-cytoplasmique de Notch (ICN) dans le noyau et une activation de la transcription des gènes cibles de RBPJ par déplacement de complexes de répression transcriptionnelle, incluant $0 \mathrm{TT}$, et recrutement de co-activateurs transcriptionnels. L'activation de la voie Notch permet l'engagement des progéniteurs hématopoïétiques dans la différenciation lymphocytaire $T$ ou dans la différenciation mégacaryocytaire. Dans le contexte de la fusion OTT-MAL, l'interaction de la protéine de fusion avec RBPJ entraîne une activation anormale des gènes cibles de RBPJ mais n'est pas suffisante pour induire une transformation mégacaryoblastique chez

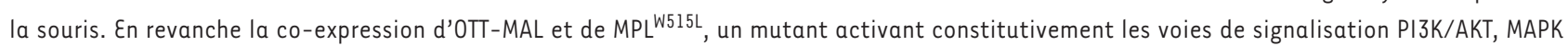
$(\varepsilon R K 1 / 2)$ et STAT, entraîne le développement de LAM7 chez la souris avec une fréquence élevée, indiquant une coopération entre ces deux oncogènes spécifiques des hémopathies mégacaryocytaires humaines. TPO : thrombopoïétine. 


\section{REMERCIEMENTS}

Nous remercions les membres des laboratoires d'Olivier Bernard et Gary Gilliland pour leur participation. Ces travaux ont été financés par l'Inserm et la Ligue nationale contre le cancer-comité de Paris (Olivier Bernard), le National Institute of Health et la Leukemia and Lymphoma Society (Gary Gilliland), et la Leukemia and Lymphoma Society et l'Inserm (Thomas Mercher).

\section{CONFLIT D'INTÉRÊTS}

Olivier Bernard et Thomas Mercher déclarent n'avoir aucun conflit d'intérêts. Gary Gilliland est devenu un employé de Merck \& Co après la publication de l'article original.

\section{RÉFÉRENCES}

1. Dastugue N, Lafage-Pochitaloff $M$, Pagès MP, et al. Cytogenetic profile of childhood and adult megakaryoblastic leukemia (M7): a study of the groupe français de cytogénétique hématologique (GFCH). Blood 2002; $100: 618-26$.

2. Duchayne $\varepsilon$, Fenneteau 0 , Pages MP, et al. Acute megakaryoblastic leukaemia : a national clinical and biological study of 53 adult and childhood cases by the groupe français d'hématologie cellulaire (GFHC). Leuk Lymphoma 2003; 44 : 49-58.

3. Wechsler J, Greene M, McDevitt MA, et al. Acquired mutations in GATAl in the megakaryoblastic leukemia of Down syndrome. Nat Genet 2002 ; 32 : 148-52.

4. Mercher T, Coniat MB, Monni R, et al. Involvement of a human gene related to the Drosophila spen gene in the recurrent $t(1 ; 22)$ translocation of acute megakaryocytic leukemia. Proc Natl Acad Sci USA 2001; 98 : 5776-9.

5. Raffel GD, Mercher T, Shigematsu H, et al. Ottl (Rbml5) has pleiotropic roles in hematopoietic development. Proc Natl Acad Sci USA 2007 ; 104: 6001-6.
6. Ma X, Renda MJ, Wang L, et al. Rbml5 modulates Notch-induced transcriptional activation and affects myeloid differentiation. Mol Cell Biol 2007; 27: 3056-64.

7. Miralles F, Posern G, Zaromytidou Al, Treisman R. Actin dynamics control SRF activity by regulation of its coactivator MAL. Cell $2003 ; 113$ : 329-42.

8. Mercher T, Raffel GD, Moore SA, et al. The OTTMAL fusion oncogene activates RBPJ-mediated transcription and induces acute megakaryoblastic leukemia in a knockin mouse model. J Clin Invest $2009 ; 16: 852-64$

9. Mercher T, Cornejo MG, Sears C, et al. Notch signaling specifies megakaryocyte development from hematopoietic stem cells. Cell Stem Cell 2008; $3: 314-26$.

10. Wilson A, Radtke F. Multiple functions of Notch signaling in self-renewing organs and cancer. FEBS Lett $2006 ; 580: 2860-8$.

11. Kastner P, Chan S. La voie Notch au centre du mécanisme de leucémogenèse dans un modèle murin de leucémies T. Med Sci (Paris) 2006 ; 22 : 708-10.

12. Debili $\mathrm{N}$, Vainchenker W. De macro à micro : I'histoire de la plaquette. Med Sci (Paris) 2008 ; 24 : 467-9.

\section{NOUVELLE}

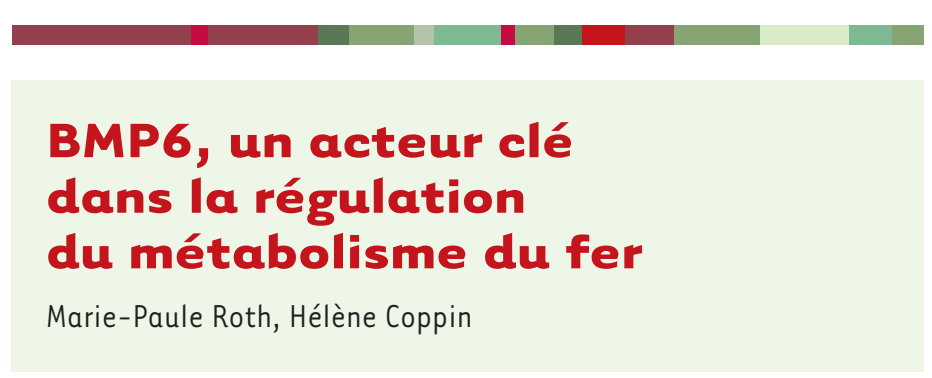

> L'absorption par la muqueuse intestinale du fer contenu dans le bol alimentaire constitue la seule voie d'entrée de ce nutriment dans l'organisme. Le fer est capté par les cellules épithéliales du duodénum, transféré du pôle apical des entérocytes vers le pôle basal qu'il traverse grâce à la ferroportine pour passer dans la circulation sanguine. Les pertes en fer sont limitées dans les conditions physiologiques et sont principalement dues à l'élimination des cellules intestinales et cutanées sénescentes. L'homéostasie du fer dépend donc essentiellement de la régulation de l'entrée du fer dans l'organisme [1].

La découverte de deux acteurs clés dans l'adaptation de l'absorption intestinale du fer aux besoins de l'organisme, l'hepcidine en 2001 [2, 3] et tout récemment la molécule qui régule sa production, BMP6 $[4,5]$, permet désormais de mieux comprendre les mécanismes permettant le maintien de l'homéostasie du fer [11, 12].

\section{L'hepcidine et la régulation du fer}

L'hepcidine se fixe à la ferroportine à la surface des entérocytes et des macrophages. Elle provoque ainsi l'internalisation et la dégradation de la ferroportine [6], bloquant le fer dans ces cellules et le rendant indisponible. Dans le foie, la transcription du gène codant l'hepcidine (HAMP) est ajustée aux besoins de l'organisme. Dans les conditions physiologiques, le niveau d'hepcidine augmente lorsque le fer est en excès et au contraire diminue en cas de carence en fer ou d'hypoxie. Cette régulation ne fonctionne plus de façon appropriée chez les patients atteints d'hémochromatose, une maladie géné-
Inserm U563, CHU Purpan, place du Docteur Baylac, 31300 Toulouse, France. marie-paule.roth@inserm.fr

tique qui conduit à une accumulation de fer, principalement dans le foie, et à des complications parfois sévères (cirrhose, hépatocarcinome). Les hémochromatoses à début précoce sont dues à des mutations dans le gène HAMP ou dans le gène HFE2, codant l'hémojuvéline, et sont plus sévères que les formes à début plus tardif dues à des mutations dans d'autres gènes impliqués dans la voie de régulation de l'hepcidine, comme HFE ou TFR2 (transferrin receptor 2).

\section{La voie de signalisation BMP-SMAD}

La mise en évidence du rôle de l'hémojuvéline (HJV ou RGMc), la protéine impliquée dans l'hémochromatose juvénile, a permis une avancée significative dans l'élucidation de la cascade de régulation conduisant à la production d'hepcidine. Il s'agit en effet d'une protéine 